\title{
Derivation of the Fundamental Missile Guidance Equations
}

\author{
PAPP István, ${ }^{1}$ SZILVÁSSY László, ${ }^{2}$ ÓVÁRI Gyula ${ }^{3}$
}

\begin{abstract}
In order to guide itself to a successful target intercept, the missile must obtain in-formation about the target. The prelaunch and post launch information too, must be gathered. Before a missile is launched, that is, during the prelaunch phase, the missile needs to know where to go. It knows that it is supposed to go to the target, but it must be told where the target is. The missile is told where the target is by electrical signals entering through the umbilical from the launcher. These signals are head aimed (to point the missile head at the target), English bias (to point the missile at the intercept point), and an estimate of true target Doppler on the simu-lated Doppler line.
\end{abstract}

Keywords: missile, equations of motion, guidance, derivation

\section{Introduction}

Then missile then flies (according to the proportional navigation guidance law), senses a change in the line-of-sight angle between the missile velocity vector and the target. In addi- tion, the missile is given certain conditioning signals, which let the missile adjust for varia- tions. These conditioning signals are the autopilot commands to adjust autopilot responses, and auxiliary Doppler positioning signal (or signals). Furthermore, though the missile is de-signed to guide to impact, an actual impact may not occur, and the missile may miss the target by some finite distance. Specific circuits in the missile give an indication of closest missile approach to the target. These circuits then cause the warhead to be triggered so as to explode as close to the target as possible. In addition, other circuits in the missile are designed to provide indications of a total miss. All of this logic and information is built into the missile, so that the missile knows what to do before it is launched. [1]

\section{Missile Guidance Types}

Guidance systems can use any one of several methods or laws to coordinate a missile along a trajectory or flight-path to intercept a target (for example an aircraft). The specific target flight path information required by the guidance package depends on which law is used. In this section we will discuss the three types of pursuit courses, which are the following:

- pure pursuit;

- deviated pursuit;

- pure collision.

\footnotetext{
National University of Public Service, Budapest, Hungary, e-mail: pappi@uni-nke.hu

National University of Public Service, Budapest, Hungary, e-mail: szilvassy.laszlo@uni-nke.hu

National University of Public Service, Budapest, Hungary, e-mail: ovary.gyula@uni-nke.hu
} 

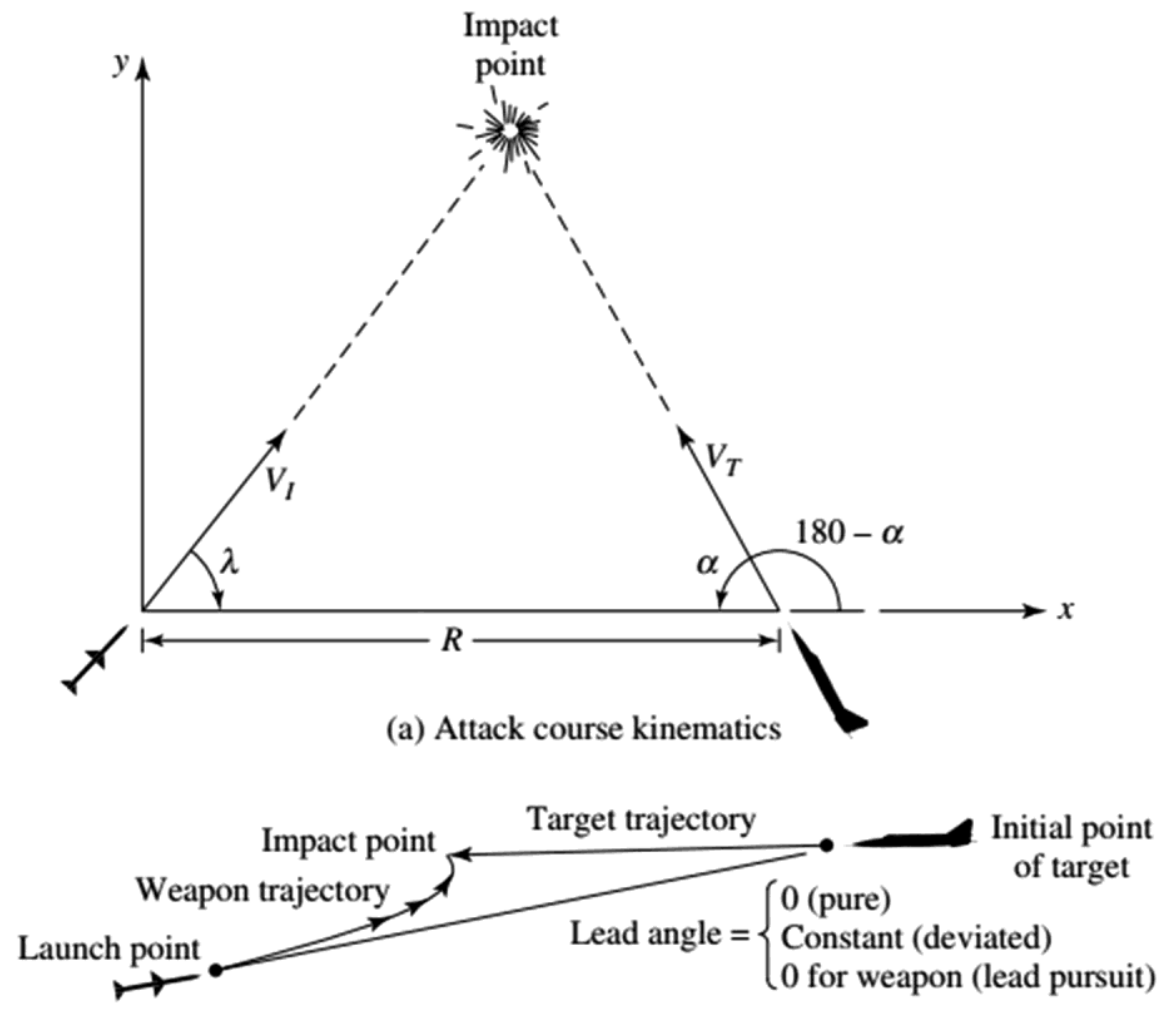

(b) Pursuit: pure pursuit/deviated pursuit/lead pursuit

Figure 1. Types of the guidance. [1: 183]

After that, we develop the respective differential equations. The homing trajectory that a missile flies depends on the type of guidance law employed. The guidance law depends on the mathematical requirements or constraints of the engagement. Figure 1 will be used as the basis to derive these equations. In particular, the kinematics of an attack course, as illustrated in Figure 1, are based on the relationships between the:

- interceptor (or missile) velocity $V_{\dot{p}}$

- the target velocity $V_{T}$;

- the interceptor lead angle $\lambda$;

- the target aspect angle $\alpha$;

- the interceptor to target range $R$.

The basic differential equations can be derived from considerations of the geometry. Referring to Figure 1, the range rate can be written in the form

$\frac{d R}{d t}=V_{1} \cos \lambda+V_{T} \cos (180 \alpha)=V_{1} \cos \lambda-V_{T} \cos \alpha$

where the angle reference is the interceptor-to-target range vector. The velocity compo- nents orthogonal to $R$ consist of two parts:

(1) the translational component, and

(2) the tangential (or turning) component. 
Selecting the interceptor as the reference point for the tangential component, and taking $d \lambda / d t$ positive in the same sense as $\lambda$, the equations can be written:

$R\left(\frac{d \lambda}{d x}\right)=V_{I} \sin \lambda+V_{T} \sin (180-\alpha)=V_{I} \sin \lambda-V_{T} \sin \alpha$

The conditions for the various types of trajectories result from holding constant one of the parameters in the equations. [1]

\section{Pure Pursuit}

In the pure pursuit trajectory, the interceptor missile flies directly toward the target at all times. Thus, the heading of the missile is maintained essentially along the line of sight be- tween the missile and the target by the guidance system. Missiles flying a pure pursuit course usually end up in a tail-chase situation, similar to a dog chasing a rabbit. Furthermore, in pure pursuit the nose of the interceptor missile (note that the term interceptor is used to denote missiles as well as fighter aircraft) is pointed at the target aircraft. The interceptor missile directing its velocity vector toward the target flies a pure pursuit attack course. In such a case the interceptor's lead angle is zero. Examine now Figure 2.

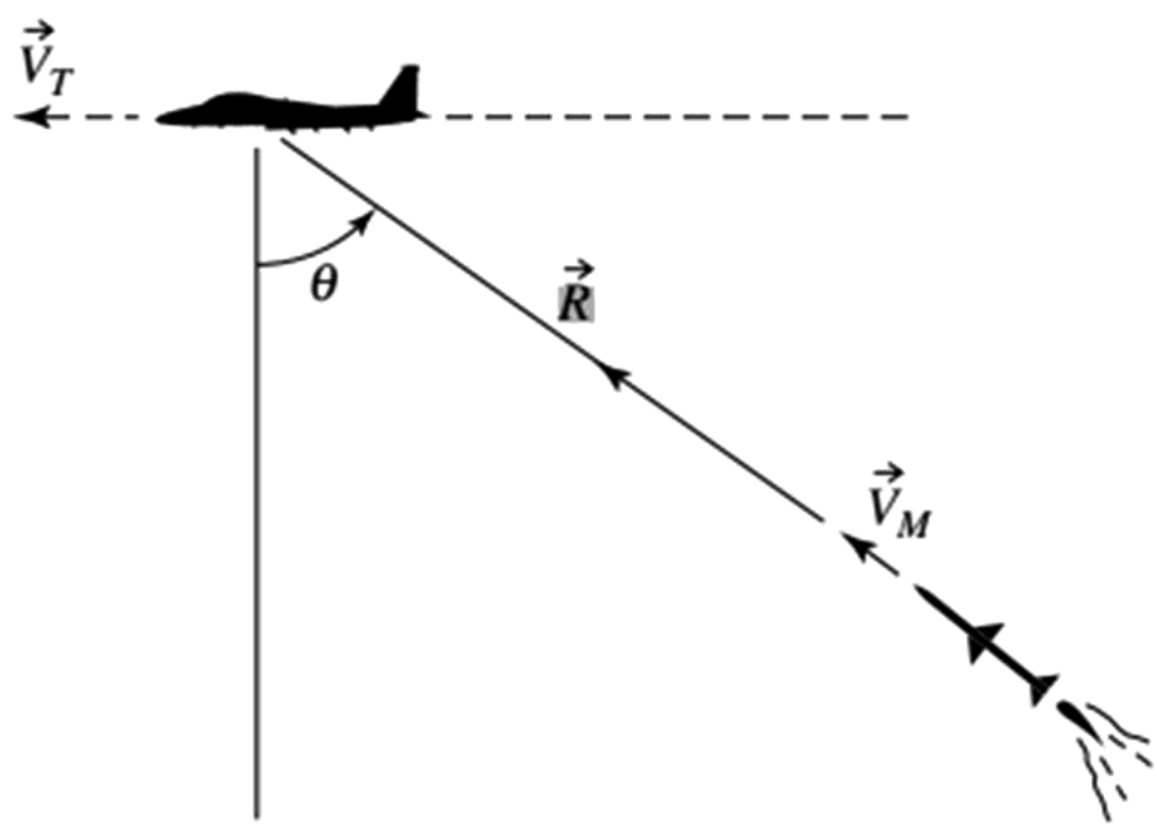

Figure 2. Pure pursuit guidance. [1: 184]

Based on the Figure 2, the following equations can be written:

$\frac{d R}{d t}=V_{M}-V_{T} \cos \theta$

(3a). (3b).

$R\left(\frac{d \theta}{d t}\right)=-V_{T} \sin \theta$ 
Where $R$ is the range magnitude, $\theta$ is the orientation of the line of sight to the target, $V_{M}$ is the interceptor missile velocity component, and $V_{T}$

nontrivial cases of a stationary target or head/tail chase $\left(\theta= \pm 90^{\circ}\right)$, we have

$$
\begin{aligned}
& \left(\frac{d \theta}{d t}\right) / R=\left\{(\cos \theta)\left(\frac{V_{M}}{V_{T}}\right)+\tan \theta\right\}\left(\frac{d \theta}{d t}\right)=\left\{\left(\frac{\kappa}{\cos \theta}\right)+\tan \theta\right\}\left(\frac{d \theta}{d t}\right) \\
& \text { where } \kappa=V_{M} T \\
& \int\left(\frac{d R}{R}\right)=\int \tan \theta d \theta+\kappa \int\left(\frac{d \theta}{\cos \theta}\right)
\end{aligned}
$$

Letting $C$ be the constant of integration, the general solution of (5) assumes the form

$\ln (R / C)=-\ln \cos \theta+(\kappa / 2) \ln [(1+\sin \theta) /(1-\sin \theta)]$

Therefore,

$R / C=(1 / \cos \theta)[(1+\sin \theta) /(1-\sin \theta)]^{\kappa / 2}$

From the identity

$1 / \cos \theta=1 /(1+\sin \theta)^{1 / 2}(1-\sin \theta)^{1 / 2}$

We have

$R / C=\rho=\left[(1+\sin \theta)^{(\kappa-I) / 2}\right] /\left[(1-\sin \theta)^{(\kappa+1) / 2}\right]$

The integration constant $C$ can be determined from the initial conditions $\mathrm{R} 0$ and $\theta 0= \pm$ $90^{\circ}$. Thus from (6) we obtain

$$
\begin{aligned}
& \lim _{0 \rightarrow 90^{\circ}} R=\infty, \\
& \theta=0, \rho=1, R=C . \\
& \lim _{0 \rightarrow 90^{\circ}} R= \begin{cases}0, & \text { when } \kappa>1, \\
\frac{R,}{2} & \text { when } \kappa=1, \\
\infty, & \text { when } \kappa<1 .\end{cases}
\end{aligned}
$$

From the above analysis, we note that the missile will intercept the target if its velocity is greater than that of the target. From (6), $\rho(\theta)$ can be plotted for different values of the param- eter $\kappa$ (i.e., $\kappa=0.5,1.0,1.5,2.0,3.0)$. [1] [2]

\section{Deviated pursuit}

Deviated pursuit points the nose of the intercepting missile (or aircraft) by a fixed angle in front of the target (see Figure 1). In other words, an interceptor directing its velocity vector at a constant angle ahead of the target flies a deviated pursuit attack course. Since the inter- ceptor lead angle is constant for deviated pursuit, $\lambda=\lambda$. Therefore, from (1) and (2) we have the differential equations

$$
\begin{aligned}
& \frac{d R}{d t}=V_{1} \cos \lambda_{0}-V_{T} \cos \alpha \\
& R\left(\frac{d \lambda}{d t}\right)=V_{1} \cos \lambda_{0}-V_{T} \cos \alpha
\end{aligned}
$$




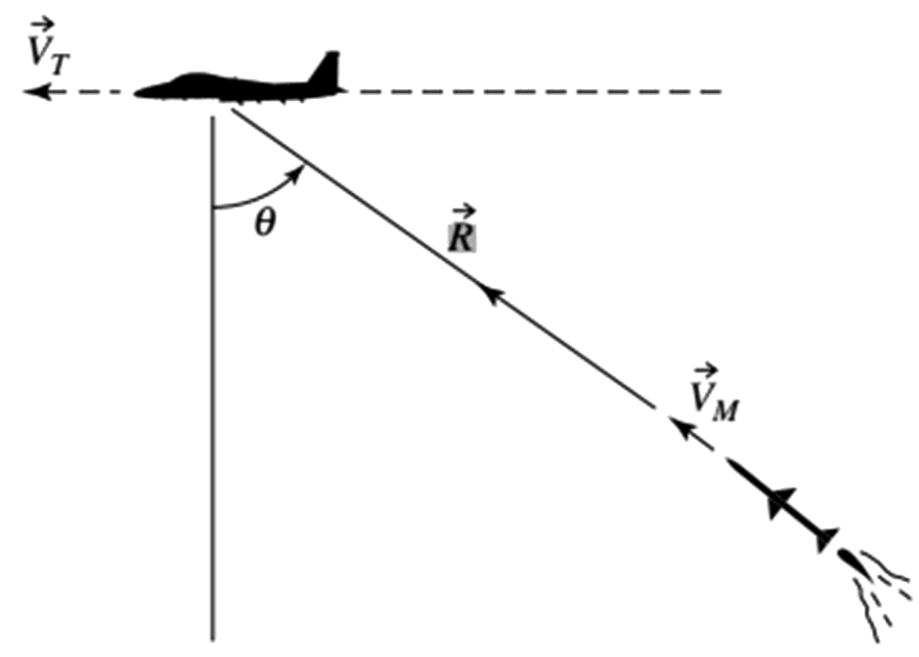

Figure 3. Deviated pursuit guidance. [1: 186]

In order to obtain the deviated pursuit algebraic equations, we will use Figure 3. The differential equations for the deviated pursuit case are $(\lambda=$ constant $)$

$\frac{d R}{d t}=-V_{M} \cos \lambda+V_{T} \cos \theta$

$R\left(\frac{d \theta}{d t}\right)=V_{M} \sin \lambda-V_{T} \sin \theta$

Solution of the differential equations for $\mathrm{R}$ and $\theta$ requires a given $\mathrm{V}_{\mathrm{M}}$

initial values of $\mathrm{R}$ and $\theta$. The normal acceleration for the deviated pursuit is obtained as

$a_{n}=-V_{M} \theta / g=\left(V_{M} / g R\right)\left[V_{T} \sin \theta-V_{M}\right]$

The angle off the target tail, at which $\theta$ is maximum, is obtained from the expression

$\theta(\max g)=\cos ^{-1}\left[V_{M} / 2 V_{T}\right]$

where $V_{M}$

intercept the target can be obtained from the expression

$t=\left(1 / V_{T}\right) \int R d \theta /\left[\left(V_{M} / V_{T}\right) \sin \lambda-\sin \theta\right]$

\section{Pure collision}

A pure collision course is a straight-line course flown by an interceptor so as to collide with the target. Referring to Figure 4.9, the differential equation assumes the form

$$
\begin{aligned}
& \frac{d R}{d t}=-V_{M} \cos \lambda+V_{T} \cos \theta \\
& \theta=\text { konstans } \\
& \lambda=\sin ^{-1}\left(V_{T} \sin \theta / V_{M}\right) \\
& R=R_{0}+\left(\frac{d R}{d t}\right) t
\end{aligned}
$$


In addition to the three guidance courses just discussed, another course of interest is the lead collision course. A lead collision course is a straight-line course flown by the interceptor such that it will attain a single given firing position. For lead collision, the time of flight (a derived parameter) is constant. Generation of this course is begun by specifying $V$, $V, V$,

$t_{f}$ and the initial angle $\theta_{0}$. The differential equations for lead collision can be obtained in a straightforward manner from Figure 3 as

$$
\begin{aligned}
& \frac{d R}{d t}=-V_{M} \cos \lambda+V_{T} \cos \theta \\
& R\left(\frac{d \theta}{d t}\right)=V_{M} \sin \lambda-V_{T} \sin \theta
\end{aligned}
$$

where

$$
\begin{aligned}
& \lambda=\sin ^{-1}\left\{-R\left(\frac{d \theta}{d t}\right) t_{g} / V_{0} t_{f}\right\} \\
& t_{g}=\left(-R+V_{0} t_{f} \cos \lambda\right) /\left(\frac{d R}{d t}\right), t_{f}=\text { konstans }
\end{aligned}
$$

Note that collision courses are flown so as to cause the interceptor missile or aircraft to collide with the target. [3]

From the guidance techniques discussed above, the two most popular techniques are pure pursuit and proportional navigation. However, proportional navigation is more complicated to mechanize in terms of hardware, whereas pure pursuit causes higher aerodynamic loading on the airframe. The basic difference between the two is that pursuit guidance causes the in- terceptor missile to home on the target itself, while proportional navigation guidance causes the missile to home on the expected impact point. No matter which method is selected by the missile designer, in order to achieve a target kill the missile must be able to pull sufficient g's to intercept the target within the lethal distance of the warhead. At lower altitudes, the airframe is not a limiting factor because the available g's are in excess of the autopilot limit (e.g. $25 \mathrm{~g})$. At higher altitudes, especially in a snap-up attack, available g's are usually the parameter that determines the launch boundary. [3]

The maximum possible missile turn rate is a limiting factor at minimum range. This is because in a minimum-range situation, an air-to-air missile is usually required to turn rapidly to intercept the target within the short flight times. The maximum turn rate of the missile is limited by two factors:

1. autopilot saturation,

2. maximum wing deflection.

The pitch or yaw autopilot will saturate when the corresponding commanded lateral ac- celeration exceeds, for example, $25 \mathrm{~g}$ 's. This is predominant at low altitudes, where the mis- sile maneuver is not aerodynamically limited. At high altitudes, the wing deflection required for turning increases, and its maximum value becomes the limiting parameter. When either type of limiting occurs, miss distance increases very rapidly. [1] [3] 


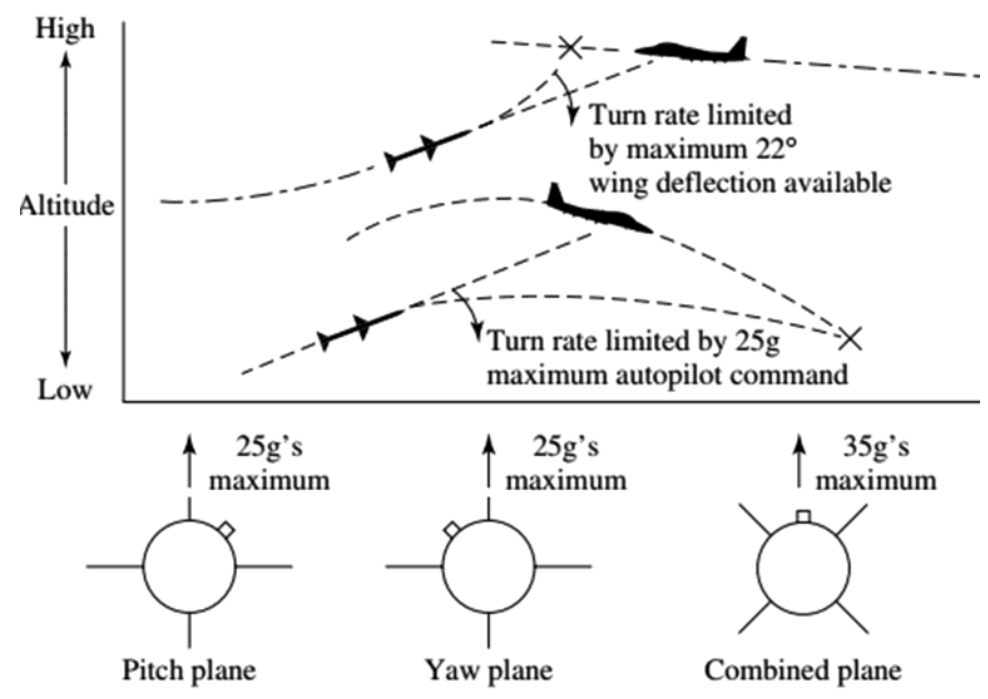

(a) Maximum maneuver capability.

Figure 4. Maximum manoeuvre capability. [1: 190]

The other factor influencing maximum turn rate is the roll orientation of the missile with respect to the manoeuvre plane. If the direction of the turn is perpendicular to either the pitch or yaw plane, then the turn will be confined only to that plane, and the maximum acceleration will be limited by the autopilot to $25 \mathrm{~g}$ 's. If the direction of the turn is halfway between the two planes, both autopilots will contribute, and the allowable turning acceleration is as high as $25 \sqrt{ } 2$, i.e., about 35 g's. That the time for which the missile locks on the target can vary from about 0.6 to 1.0 second. Increased lock time can have a significant affect because of the rapidly changing geometry, and usually results in increased missile flight times to attain a successful intercept. Because lock time is an uncontrollable factor, a degree of uncertainty is introduced to the minimum-range zone. These relationship, we can see in Figure 4. [1] [4]

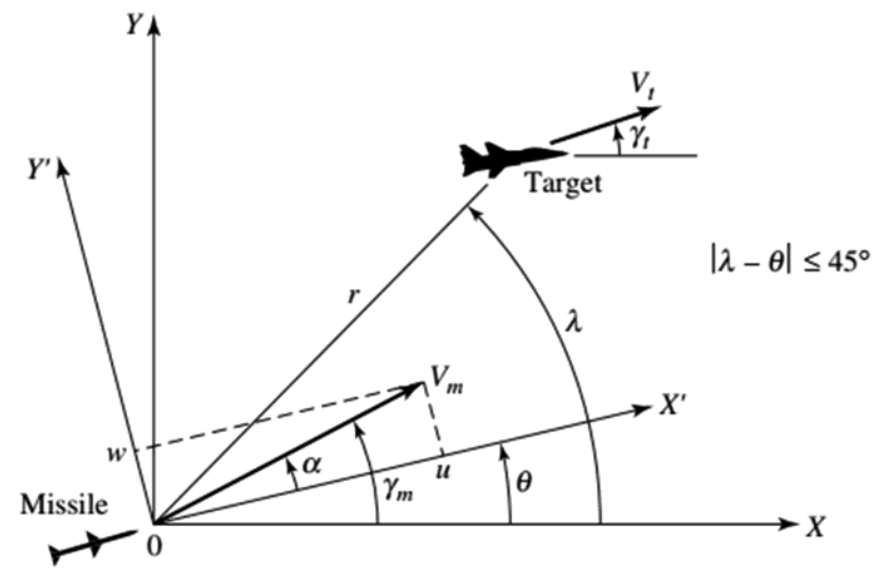

(b) Geometry of the interception process.

Figure 5. Geometry of the interception process. [1: 190] 
At this point, simple interception model dynamics will be developed. Assuming that the target and the missile motions evolve in the same horizontal plane, the geometry of the in- terception process is shown in Figure 5. The interception is characterized by two variables, namely, the target range and the LOS angle. The kinematic equations are expressed by the following relations:

$$
\begin{aligned}
& \frac{d r}{d t}=V_{t} \cos \left(\lambda-\gamma_{t}\right)-u \cos (\lambda-\theta)-\omega \sin (\lambda-\theta), \\
& \frac{d \lambda}{d t}=-\left[V_{t} \sin \left(\lambda-y_{t}\right) \sin (\lambda-\theta)-\omega \cos (\lambda-\theta)\right] / r, \\
& \text { where } \\
& r=\text { missile - target range; } \\
& \lambda=\text { LOS angle; } \\
& \theta=\text { pitch angle (or missile axis angle); } \\
& u=\text { longitudinal velocity component of the missile; } \\
& w=\text { normal velocity component of the missile. }
\end{aligned}
$$

The rate of variation of the $L O S, d \lambda / d t$, is measured by the seeker, and the tracking error related to the system of measurement is neglected. In other words, the axis of the seeker is assumed to lie always along the LOS. The seeker head will be limited to a cone with a maxi- mum half-angle equal to $45^{\circ}$, which imposes the saturation constraint $|\lambda-\theta| \leq 45^{\circ}$. [1] [3] [4]

\section{References}

[1] SIOURIS, G. M.: Missile Guidance and Control Systems. New York: Springer, 2003. http:// cdn.preterhuman.net/texts/terrorism_and_pyrotechnics/rocketry/Missiles_and_Warheads/ Missile\%20Guidance\%20\&\%20Control\%20Systems.pdf (downloaded: 2015.07.15)

[2] BALL, R. E.: The Fundamentals of Aircraft Combat Survivability Analysis and Design. Virginia: Virginia Polytechnic Institute and State University Blacksburg, 1985.

[3] PALUMBO, N. F., BLAUWKAMP, R. A., LLOYD, J. M.: Modern Homing Missile Guidance Theory and Techniques. Johns Hopkins APL Technical Digest, 291 (2010).

[4] LIN, C. M., HSU, C. F., CHANG, S. K., WAI, R. J.: Guidance - Law Evaluation for Missile Guidance Systems. Asian Journal of Control, 24 (2000), 243-250. DOI: https://doi.org/10.1111/j.1934-6093.2000.tb00029.x 